This is a pre-peer reviewed version of the following article: Singh, S. P.,

McCartney, M., Singh, J. and Clarke, R. (2008), RFID research and testing for

packages of apparel, consumer goods and fresh produce in the retail distribution

environment. Packaging Technology and Science, 21: 91-102. doi: 10.1002/pts.782,

which has been published in final form at http://onlinelibrary.wiley.com/doi/10.1002/pts.782/abstract

\title{
RFID Research and Testing for Packages of Apparel, Consumer Goods and Fresh Produce in the Retail Distribution Environment
}

S. P. Singh

Michigan State University, East Lansing, Michigan, USA

M. McCartney

QLM Consulting, Sausalito, CA, USA

\section{J. Singh}

Cal Poly State University, San Luis Obispo, CA, USA

\section{R. Clarke}

Michigan State University, East Lansing, Michigan, USA

\section{ABSTRACT}

Radio frequency identification (RFID) is a term used for any device that can be sensed at a distance by radio frequencies with few problems of obstruction. The origins of the term lie in the invention of tags that reflect or retransmit a radiofrequency signal. According to a recent article by Forrester Research, the minimal 'Slap and Ship’ approach to RFID compliance will cost an individual company between $\$ 2$ million and \$20 million. Because retailers like Wal-Mart plan to share with their suppliers all the RFID-generated data points (from when a case/pallet enters their distribution centre until it leaves their stockroom), suppliers will eventually be able to use this data as a powerful forecasting tool. RFID is an enabling technology that can potentially facilitate a realtime, end-to-end supply chain visibility system. Suppliers who integrate full-scale RFID systems will realize efficiencies in time, material movement, inventory planning, shipping and warehousing both internally and externally. This paper provides a brief overview of the RFID technology, mandates by retailers and federal 
agencies, advances towards global standardization and typical consumer level RFID applications, and discusses RFID initiatives taken by some of the global leaders in apparel, consumer goods and fresh produce industries.

KEY WORDS: radio frequency identification; smart packaging; tracking; supply chain tracking

\section{INTRODUCTION}

Radio frequency identification (RFID), a means of storing and retrieving data through electromagnetic transmission to a radio frequency (RF)- compatible integrated circuit, has been ranked the 10th most innovative technology of the past 25 years by CNN ${ }^{2}$ RFID technology presents an odd paradox; it has been around for over 60 years and yet it still stays ahead of its time. The total market, including systems and services, is predicted to reach $\$ 26.9$ billion by the year $2015 .{ }^{3}$ According to Erik Michelsen, ABI Research's director of RFID research, 'We are seeing companies increase their RFID budgets three to five times this year compared to $2004^{\prime}$. $^{4}$

RFID promises a slew of benefits to manufacturers and retailers alike, including more control over the supply chain and enhanced product security. High-profile compliance mandates [Wal-Mart, Department of Defense (DoD), etc.] and cost savings for businesses are the main factors fueling the growth of RFID. Research by the Yankee Group, an Independent Technology consultancy in Boston, indicates RFID technology could save \$2-4 billion annually for packaged consumer goods and retail industries. ${ }^{5} \mathrm{~A}$ study by the consulting firm Accenture shows that manufacturers could reduce their working capital requirements between 2 and $8 \%$, and reduce inventory levels even more with RFID-enabled processes. ${ }^{6}$ RFID deployment is expected to be substantial. Many market research firms estimate that there will be nearly a 10-fold increase in supply chain RFID use over the next 5 years. According to Robert Jaques of vnunet.com, in citing a report by IDTechEx, the worldwide RFID market is predicted to top $\$ 7$ billion by $2008 .{ }^{6}$ Additionally, RFID patents are rapidly being granted. By the end of 2003 , approximately 4300 RFID-related patents were granted, with more than three-fourths of them granted since 1999. ${ }^{6}$ 
RFID is a relatively immature technology as far as recent interests shown towards implementations in the supply chain systems. There is presently no single plug-and-play solution that can be adopted by all interested parties. This paper provides a brief insight into the application efforts and lessons learnt by some of the leading companies in the apparel, consumer goods and fresh produce industry.

\section{RFID BASICS}

RFID involves the use of electronic tags with integrated circuit chips that can store data. The tags, affixed to the asset, transmit their data via low-power radio waves to reading systems, which are tuned to the same frequency, enabling transactions to be recorded and tracked. A typical transmission sequence consists of a system handshake, data modulation and data encoding, and is illustrated in Figure 1. A form of RFID tags are battery powered or 'active', but 'passive' or 'backscatter' tags are slated to be more widely used. This category of tags derives all their power from the reader's signal. Nearly all RFID systems operate on one of four frequency bands, namely, low frequency $(125-134 \mathrm{kHz})$, high frequency $(13.553-13.567 \mathrm{MHz})$, ultra high frequency (UHF) $(400-1000 \mathrm{MHz})$ and microwave $(2.45 \mathrm{GHz})$. The read range typically increases with an increase in frequency. Low frequencies are commonly adopted for such applications as livestock tracking, card key and access-control. High-frequency RFID is more flexible and is utilized for applications such as baggage handling. UHF is the most widely used RFID band due to its robustness and reading range. Key applications for UHF band are materials management and supply chain tracking. Microwave band features in such applications as electronic toll collection and railroad monitoring. 


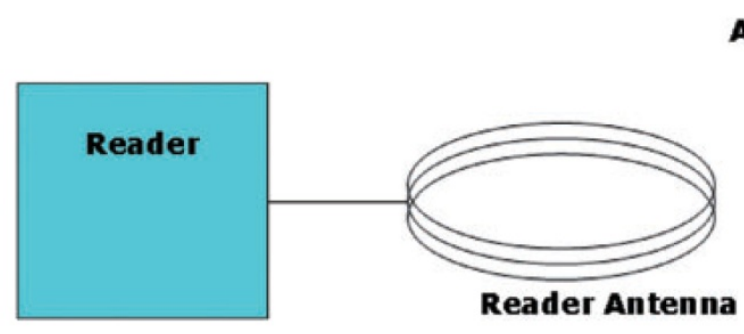

Air Interface

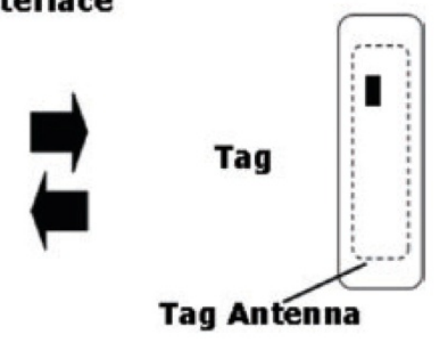

Figure 1. A typical RFID system.

Among the many advantages of RFID over other automatic identification and data capture (AIDC) technologies, such as bar codes, are often the ability to identify assets without a clear line of sight between tag and reader, ability to function in harsh environments, permit numerous tags to be read seemingly simultaneously, read/write capability and ability to provide a high level of data security. These advantages and their associated cost savings have caused some leading retailers and government agencies to issue RFID mandates to their suppliers.

\section{RFID MANDATES}

Retailers such as Wal-Mart and Target have identified RFID as a technology to help improve their supply chain management. Wal-Mart is one of the most aggressive retailers in implementing RFID. In 2004, Wal-Mart mandated its top 100 suppliers to tag all their case units and pallets delivered to three of its Texas distribution centres by 1 January $2005 .^{7}$ In spite of initial difficulties in coming to grips with the mandate, the top 100 suppliers tagged at least one stock-keeping unit (SKU) category in their shipments to Wal-Mart's distribution centres. Another 38 suppliers voluntarily decided to work with Wal-Mart to meet its RFID requirements. WalMart required its next 200 top suppliers to comply with a similar mandate by January 2006, and further 300 suppliers by January 2007. RFID provides an opportunity to reduce supply chain costs, to speed the flow of merchandise from manufacturing through distribution centres and to retail stores, and to provide consumers with better product availability. A study by the research group Gartner shows that the use of RFID in supply 
chains could result in a 90\% decrease in location errors, $40 \%$ decrease in inventory counting time and $15 \%$ increase in productivity. ${ }^{8}$ Retailers, at the present time, are requiring suppliers to provide RFID tags at case and pallet level, and eventually will move on to item-level tagging.

Government agencies, such as the DoD and the Food and Drug Administration (FDA), are also considering their suppliers to incorporate RFID tags in their shipments to them. DoD mandated all contracts issued after 1 October 2004 to apply RFID tags to all cases and pallets, and to individual high-value items ( $\$ 5000$ or more) shipped to DoD. ${ }^{9}$ Due to some forecasting problems and failure to adequately notify DoD's nearly 43000 suppliers of the RFID mandate and the current RFID tag shortage, the date was pushed back to April $2005 .{ }^{9}$ With the increasing drug-counterfeiting concerns, FDA has identified RFID as a major tool in its attempts to combat this problem. RFID is to help create a 'pedigree' (a secure record documenting that a drug was manufactured and distributed under safe and secure conditions) for drugs manufactured by pharmaceutical companies. Companies like Purdue Pharma, GlaxoSmithKline and Pfizer have already commenced pilot programs to incorporate RFID in cases of products deemed susceptible to counterfeiting.

Up until early 2006, a lack of harmonized specification (the communications protocol between RFID tags and readers) threatened to substantially delay the implementation of RFID at a global level. Vendors adopted various electronic product code (EPC) standards such as EPC Class 0, 64/96-bit read-only tags and Class 1, 64/96-bit write once-read many (WORM) tags. This led to vendor hardware incompatibility, low tag-yield rates, high tag costs and manufacturing capacity constraints. In December 2004, EPCglobal, a developer of industry standards for EPC, ratified its second generation EPC specification for tracking goods using UHF tags. The 'Gen 2' standard promises a number of much more sophisticated features than 'Gen 1' protocols. These include a global, open, interoperable standard, faster read rates (10 times faster than Gen 1), smaller size chips (approximately 20\%), high reliability, denser reader operation, kill security (enhancement with 32-bit password encryption and permanent kill capacity) and improved write capability. ${ }^{10}$ Starting as early as January 2005 (Impinj $)^{11}$ and later the 
same year (third quarter of 2005 for companies like Phillips Semiconductors and Texas Instruments), companies had or were planning to start full production of Gen 2 chips. ${ }^{12}$

\section{RFID APPLICATIONS AND INDUSTRY INITIATIVES}

RFID is being adopted in three principle areas: transportation and distribution, manufacturing and processing, and security and law enforcement. ${ }^{13}$ Secondary areas of application, some of which are steadily growing in terms of application numbers, include animal tagging, waste management, time and attendance, postal tracking, airline baggage reconciliation, and road toll management.

The supply chain at its present stage is not as reliable as it needs to be. Every year, billions of dollars are lost because products are not shipped on time or in the right quantities. Often, wrong products are shipped or the shipments get accidentally misdirected. On occasion, shipments get miscounted or miscoded on the receiving end, and sometimes, loss is created by theft, which can occur at any point in the supply chain. RFID-based supply chain management systems promise the potential to rectify a majority of the shortcomings of the present-day supply chain.

\begin{tabular}{|lc|}
\hline \multicolumn{2}{|c|}{$\begin{array}{c}\text { Table I. Estimated number of RFID tags in supply chain for } \\
\text { major consumer product companies }\end{array}$} \\
End user & $\begin{array}{c}\text { Estimated number of units in } \\
\text { supply chain (billions) }\end{array}$ \\
\hline CHEP & 0.2 \\
Johnson \& Johnson (Consumer Goods Division) & 3 \\
Kimberly Clark & 10 \\
Westvaco & 10 \\
The Gillette Company & 11 \\
Yuen Foong Yu Paper Manufacturing Co. & 15 \\
Tesco & 15 \\
The Procter \& Gamble Company & 20 \\
Unilever & 20 \\
Philip Morris Company & 25 \\
Wal-Mart & 30 \\
International Paper & 53 \\
Coca-Cola & 200 \\
Subtotal & 412.2 \\
(Adjust for double counting at I5\%) & -61.8 \\
United States Postal Services & 205 \\
Total including USPS & 555.4 \\
\hline
\end{tabular}


There are of course obstacles in the development, implementation and acceptance of RFID, as is the case with any immature technology. These obstacles include standardization, price and privacy/ ethical issues. RFID also faces challenges in cases where the product contains water-based liquids and when the tag is placed on or near metal packaging. Multinational consumer goods manufacturers such as Gillette, Kimberly Clark, and Proctor and Gamble have, in recent years, initiated RFID pilot studies to foster a new culture of innovation to achieve dramatic efficiencies in its supply chain.

On the issue of price, considering the estimated number of units in supply chain as shown in Table $1,{ }^{14}$ a universal adoption of RFID technology will provide tag manufacturers encouragement to bring down the costs with increased production. Alien Technology announced in late 2005 that their pitch labels would cost approximately 12.9 cents, and Avery Dennison is providing its inlays at 7.9 cents. ${ }^{15}$ It is clear with recent low price introductions of tags and devices by major manufacturers, these trends will continue as the use of RFID tags becomes widespread. The January 2006 issue of DC Velocity, a leading trade magazine (a publication of Agile Business Media, LLC) covering logistics solutions for distribution, predicted 900 billion food items could carry RFID tags by 2015

The next section of the paper discusses key initiatives by several companies that are supplying products and packages in the retail distribution supply chain. Several of these test results and projections were presented at the RFIDfresh and RFIDretail programmes developed by Dr. S. Paul Singh of Michigan State University and Mr. Michael McCartney of QLM Consulting in 2004 and 2005. These two programmes were attended by leading packaging, food and consumer goods companies to share results of various tests conducted to find RFID suitability to track and trace packages in the supply chain. 


\title{
CONSUMER GOODS TESTING AND EVALUATION
}

Procter and Gamble (P\&G), a global leader in consumer goods, is another company that has actively sought RFID-based solution for its supply chain. The company boasts $\$ 51.4$ billion in annual sales, approximately 300 brands in more than 160 countries and approximately 98000 employees worldwide. ${ }^{14}$

Some potential benefits that P\&G has identified for EPC adoption are improved shelf availability (sales increase), inventory reduction, labour productivity from non-line-of-sight capabilities, reducing shipping and receiving errors, overall shrink reduction, asset tracking, anti-counterfeit protection, and other future transformational benefits. The following are some of the goals P\&G has set for itself to stay ahead of the curve for RFID implementation: ${ }^{14}$

\author{
1. Form multifunctional team \\ 2. Conduct performance testing \\ 3. Incubate qualifying technology \\ 4. Evaluate validating benefits \\ 5. Stay active in EPC standards development \\ 6. Perform learning pilots
}

One of the pilots, P\&G, was involved with researched tag/reader performance optimization issues as related to four of its top-selling brand products. These included paper product (Charmin), granules (Tide powder), liquids (Pantene, Tide liquid) and metallized packages (Cascade). These product/package systems were selected to observe the readability of RFID tags through different physical constitutions. Each of these brands is prominent and recognized worldwide with sales exceeding US\$1 billion. Nine cases of the product per tier were placed three tiers high on pallets. Alien Technology's 'squiggle' tags were placed on the exposed sides of the cases as shown in Figure 2. The pallets on conveyor belts were then passed by reader antennas facing one of the sides at two different speeds, $0.625 \mathrm{mph}$ and $5 \mathrm{mph}$. 

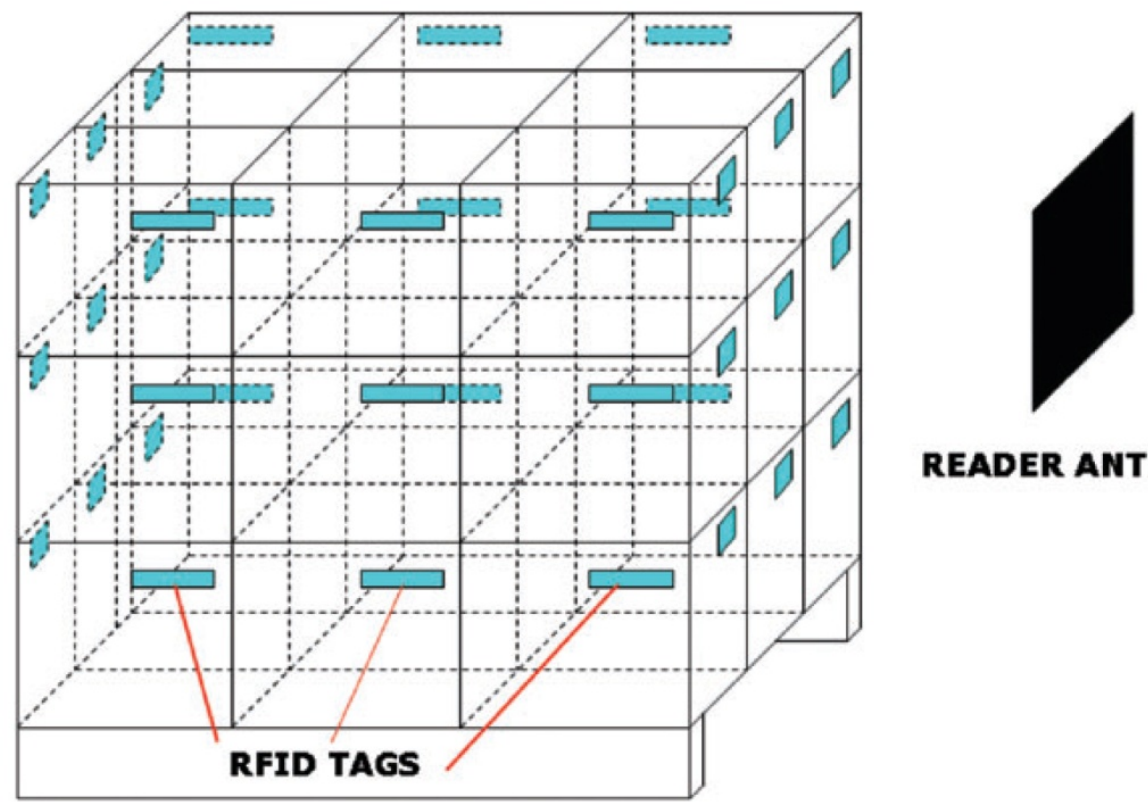

READER ANTENNA

Figure 2.Tag and reader antenna placement for palletized cases.

Some of the conclusions drawn from this pilot study and testing follows:

1. Product type is the most critical overall variable. The nature of the product and packaging material makes a significant difference in readability. Paper product packaged in a polymer film(Charmin) exhibited the best reads (near $100 \%$ at $0.625 \mathrm{mph}$ pass), followed by dry granulated powder packaged in a paperboard carton (Tide powder), liquid product in a polymer bottle (Pantene hair care product) and powdered dishwashing product packaged in an aluminum foil-laminated paperboard carton (Tide dishwashing powder).

2. The distance of the RFID tag from the circular type of reader antenna makes an impact on readability. The tags placed closest to the antenna were read more often than those farther away. Also, the tags placed at or near the height level of the reader antenna were identified better.

3. The speed at which the pallet passed by the reader antenna dictated readability of the tags. Increased pallet speed produced less reads. With the correct reader configuration and within the speeds tested, any single case in single file on a conveyor, or any single unshielded pallet tags through the dock door 
could be read. Charmin paper rolls, for example, had a near $100 \%$ readability ( $5+$ reads) at $0.635 \mathrm{mph}$ and only $20 \%$ at $5 \mathrm{mph}$.

4. The technology is not capable for auditing mixed pallets or multiple cases on a pallet, or multiple pallets through a dock door, across all product categories, against an overall objective of $100 \%$ read rate.

The results of this study are represented in Figure 3 as developed by $P \& G$ to simplify the understanding of the test results. ${ }^{14}$ This type of visualization techniques helps end-users understand the complexity of this technology and how it performs differently with different products and packages while using the same tags, hardware and software.

The P\&G Company, at the time of the presentation, was looking at the price per tag to fall to $\$ 0.05$ level in order for it to move beyond simply complying with the retailer mandates and moving forward with wider level acceptance over different products. In a presentation made by P\&G's Bud Babcock of the Customer eBusiness Division at Michigan State University's RFIDretail Seminar, ${ }^{14}$ the graph in Figure 4 was used to illustrate the impact of RFID adoption on tag costs.

Similar to P\&G and its role with consumer goods, Gillette, a century-old company, has staked out a leadership position in the use of RFID. In January 2003, Gillette placed an order of 500 million Class 1 EPC tags, the largest order ever placed. ${ }^{16}$ The goal of Gillette was to foster a new culture of innovation to achieve dramatic efficiencies in its supply chain. The company has staked out a leadership position in the use of RFID, specifically the EPC developed by the Auto-ID Centre. Another start-up company called OAT Systems provided some of the middleware needed to filter the RFID data coming from readers.16 Gillette believes that EPC technology will ultimately provide the visibility needed to enable the company to reduce its inventory levels while ensuring that its products are always at the retail store when customers want to buy them.

In 2003, Gillette launched a major EPC trial at its packaging and distribution centre for the northeastern USA located at Fort Devens, MA. ${ }^{16}$ In an effort to validate the business case for using EPC technology and to develop a 
scalable solution, the company tracked all cases and pallets of its Venus women's razors within its centre. The goal of the pilot was not to see if tags on pallets and cases can be read automatically, but rather to develop the systems and business processes needed to sustain extraordinary levels of efficiency and productivity.

At the Fort Devens facility, a subcontractor put Venus razors into packages and then into cases. The cases were stacked on a pallet, which was then transferred to the adjacent distribution centre. There, the pallet may be loaded onto a truck bound for a retailer's store or distribution centre, or its cases may be removed and stacked on other pallets to fill orders requiring less an entire pallet's worth of the same product. The facility also had a United Parcel Service conveyor for parcel deliveries and a special pack area where items used in special promotions could be boxed. ${ }^{16}$ The EPC system now lets Gillette automatically record and track every case of Venus razors in its pack centre. The system also allows Gillette to know how long a case was at the pack centre, where it was stored and when it was shipped.

By extending the pilot to a retailer, Gillette plans to be able to quantify EPC technology's ability to improve order accuracy, reduce administrative error, facilitate the investigation of problems and eliminate areas of vulnerability. Gillette has not quantified the return on investment from EPC technology yet, but here are significant business benefits the company expects to achieve: ${ }^{16}$ 
Charmin
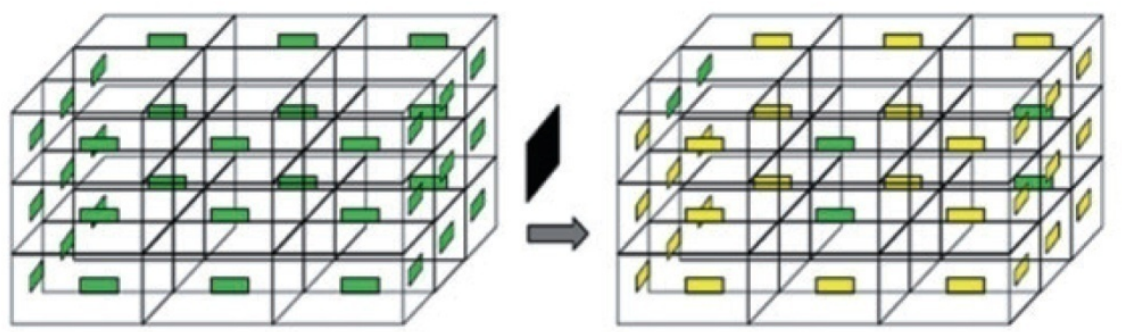

Tide Powder
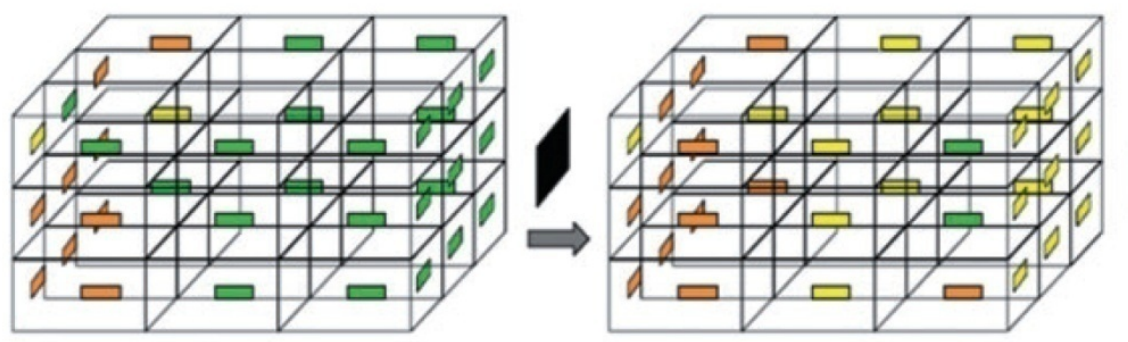

Pantene

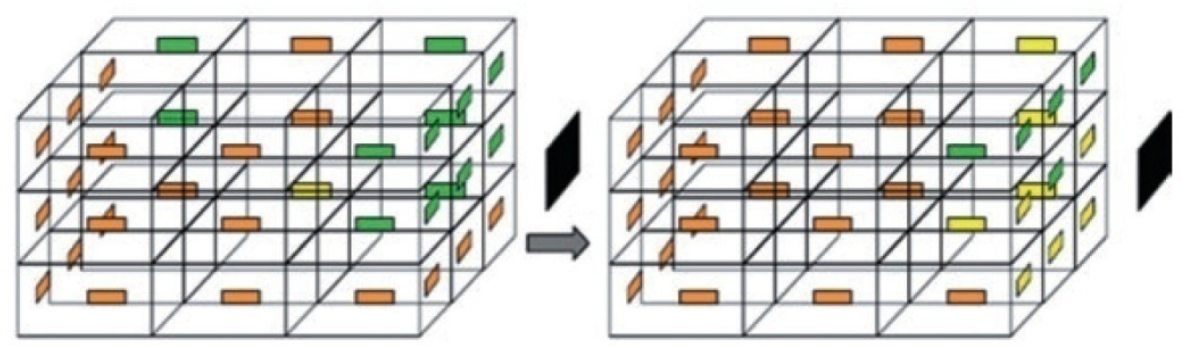

Cascade
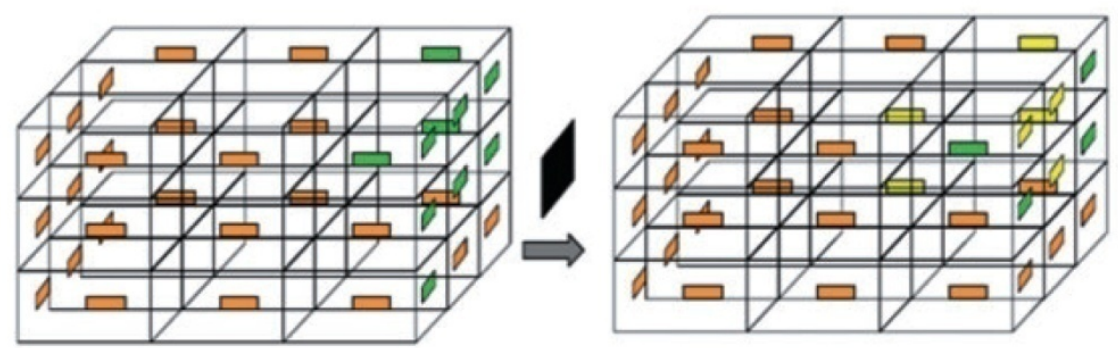

\begin{tabular}{|l|l|l|}
\hline $5+$ Reads & $1-4$ Reads & 0 Reads \\
\hline
\end{tabular}

Figure 3. P\&G tag/reader performance optimization results. ${ }^{14}$ 


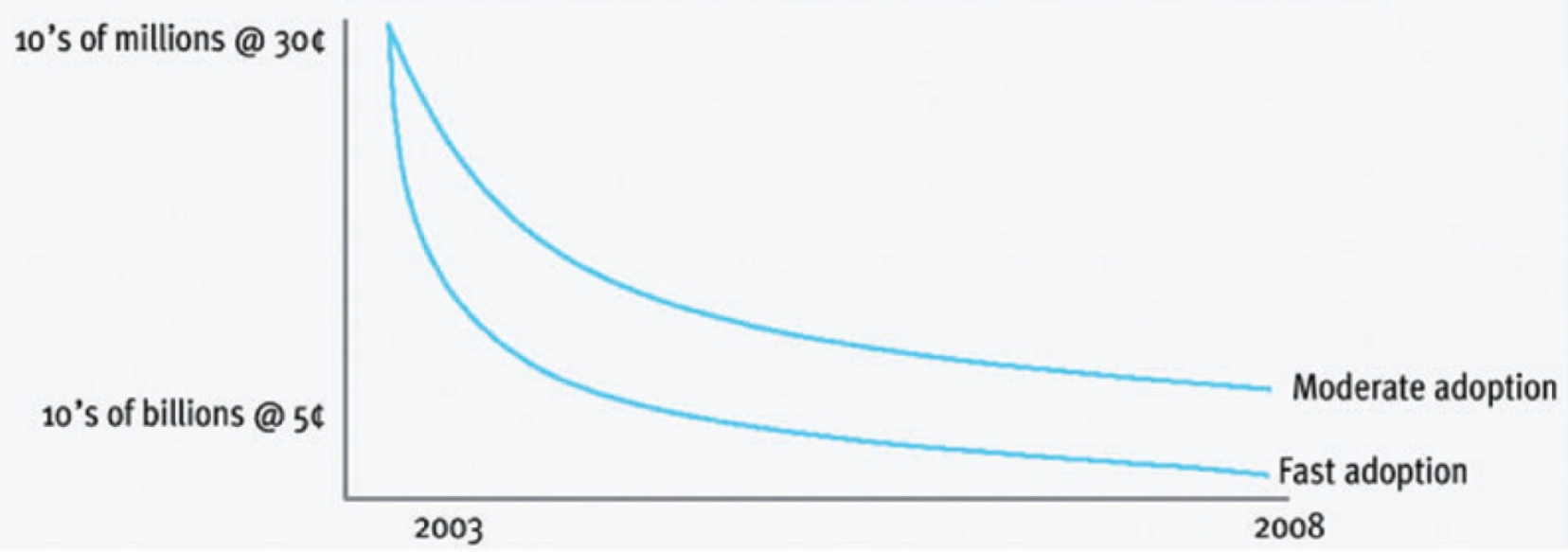

Figure 4. The impact of adoption on tag costs. ${ }^{14}$

- Reduce the number of pallet touch points, resulting in efficiencies and labour savings

- Eliminate manual case and pallet scanning

- Do away with manual case counting

- Cut back on label printing and application

- Shorten the time it takes to check an order before shipping

- Improve order accuracy

- Reduce negotiations with retailers over missing product

- Curtail shrink at distribution centres, warehouses and in transit

- Improve forecasting

- Lower overall inventory levels

- Increase on-shelf availability of products

- Improve customer service levels

\section{APPAREL PRODUCTS}


VF Corporation, an industry leader in jeanswear (Wrangler, Lee, etc.), intimates (Vanity Fair, Lily of France, etc.), outdoor wear (Jansport, Eastpak, etc.), imagewear [National Football League (NFL), Bulwark, etc.] and sportswear (Nautica) has annual sales in excess of $\$ 600$ million each year. ${ }^{17}$

VF Corporation's supply chain is very complex. It produces 500 million units annually with 500000 SKUs by over 1000 owned/contracted factories worldwide, thousands of miles away from their customers, delivered to 43 distribution centres and shipped to 47000 retailers in more than 25 countries and over 240000 households. ${ }^{17}$ All of this is done within selling seasons. For example, the NFL's Super Bowl events require that the championship team's apparel start to sell immediately after the event. Most of the sales of apparel for such special occasions peak for only weeks and not months.

To maintain better control of goods moving through its supply chain, VF Corporation started looking at RFID as a possible solution in 1993 , and developed the tag specifications by $1995 .{ }^{17}$ By 2000 , the company had identified three vendors that could meet the specifications selected. In 2000, however, the cost per tag was $\$ 0.35$, and with the required quarter to half billion tags, it far exceeded the benefits sought from such a system. ${ }^{17} \mathrm{VF}$ Corporation decided to wait for the price per tag to drop before a complete implementation of RFID for all product categories.

The goal for VF Corporation is to comply with Wal-Mart and other RFID-tagging requirements. Three VF coalitions and seven distribution centres (Figure 5) are presently involved in Wal-Mart RFID-tagging implementation. All Warehouse Management Systems (WMS) programming changes to support RFID are complete (each coalition has a separate WMS. The WMS are currently being modified to support 96-bit tags ('Gen 2'). VF jeanswear is also, presently, participating in the target RFID pilot. ${ }^{17}$ 


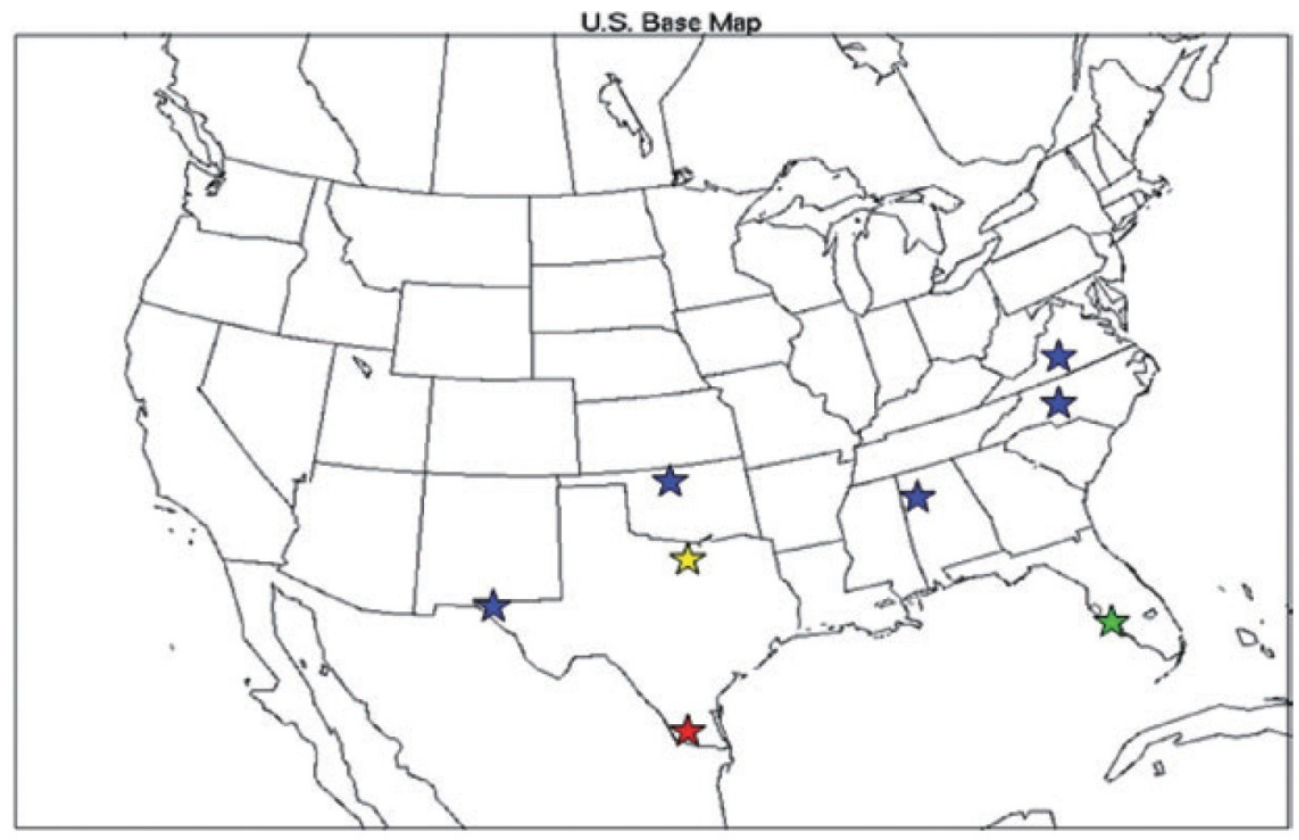

Figure 5.VF supply chain serving Wal-Mart. ${ }^{17}$

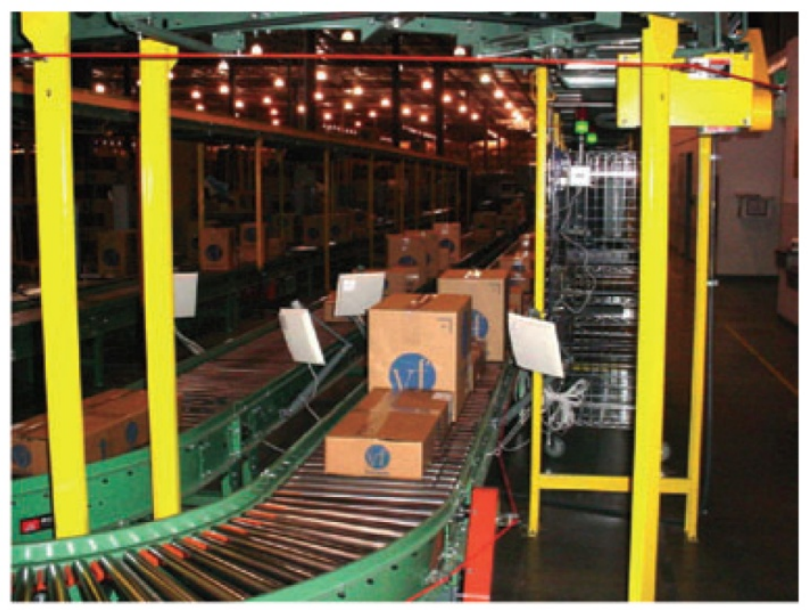

Figure 6.VF Corporation's RFID set-up in Mission, TX Distribution Center. ${ }^{17}$

By February 2005, VF Corporation had sent approximately 10000 tagged cases to Wal-Mart. Figure 6 shows VF Corporation's RFID set-up at their distribution centre in Mission, TX. Due to failure at encoding and validation, the company is currently experiencing a $5 \%$ failure rate. All of these failures are remediated before shipment to Wal-Mart. Preliminary results from Wal-Mart have shown that they are reading $98 \%$ of the tags from VF Corporation. 
Among the future RFID goals for VF Corporation are ${ }^{17}$

1. Tactical compliance with customer initiatives

2. Utilization of RFID information from Wal-Mart and other retailers

3. Strategic migration to item-level tagging including

- Internal inventory control

- Replacement of electronic article surveillance (EAS) tag

- Quality control

- Product returns

4. Procurement of tags at a realistic charge

\section{FRESH FRUITS AND VEGETABLES (PRODUCE)}

A significant amount of research was conducted at the Fresh Produce RFID Test Centre in Salinas, CA in 2004-

2005. These projects were carried out on a collaborative basis by CH Robinson Inc., Michigan State University School of Packaging, Newstar Inc., QLM Consulting Inc. and additional growers of fresh produce. In addition, during RFIDretail and RFIDfresh, Tanimura and Antle Inc., a global leading provider of fresh vegetables (cauliflower, broccoli and celery), shared some key results. The direct conclusions of the tests indicated

1. Ninety-five to one hundred per cent reads of RFID-tagged cases feasible

2. Effect of metal (forklift effect) was critical in readability

3. Effect of high water-content products, such as celery, are deterrent to RFID reads

4. Speed of travel on either conveyors for cases or for trucks with palletized loads is critical

Figure 7 shows a palletized load of fresh produce in reusable plastic containers (RPCs) tested with RFID tags. The choice of packaging material (plastic RPCs versus corrugated shipping boxes or trays) for the same produce may produce different results. Figure 8 shows a palletized load of fresh produce in corrugated boxes being tested 
through an RFID portal before being loaded into a truck through a dock door. Similarly, Figures 9 and 10 show additional variables, like the use of ice slurry and forced air cooling, which could play a role on final readability of RFID-tagged cases.

Based on these studies, McCartney ${ }_{18}$ has identified various additional factors that can play a role on final readability of RFID-tagged cases as follows:

1. Presence of external electromagnetic fields in the monitoring environment

2. Material-handling equipment (conveyors, fork trucks, pallet jacks)

3. Speed of case or pallet transfer

4. Orientation of the RFID tag/label on the shipping case or pallet

5. Unitization method such as stretch wrap, banding, etc.

6. Orientation of the RFID tag on the case to the reader

7. Case configuration on the pallet (highest reads occur when all tags are outwardly facing)

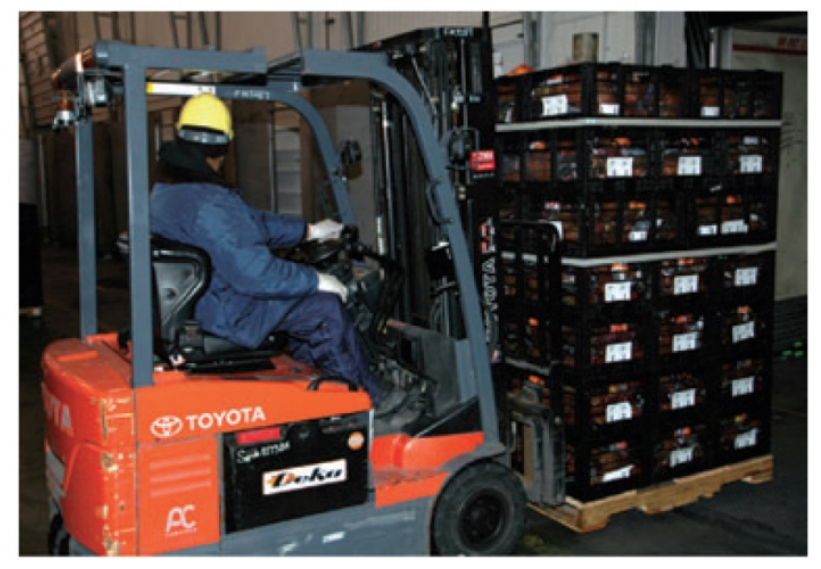

Figure 7.Testing of fork truck palletized handling of RFID-tagged reusable plastic crates. 


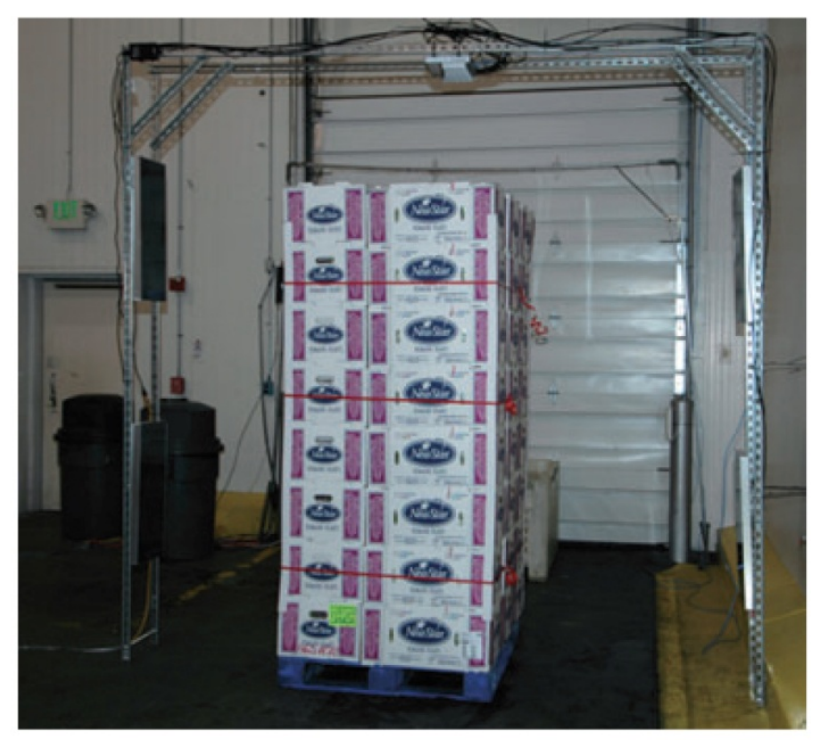

Figure 8.Testing of palletized RFID-tagged corrugated boxes in portal.

In addition, he has proposed that there are complex interactions between package configuration, type and size of packaging materials and containers, physical and climatic environmental conditions, use of material-handling equipment, the built environment of the facility, and additional electromagnetic interference from neighboring equipment. Additional factors that effect fresh produce as compared to other products are

1. Proximity of produce to the packaging container sidewall

2. Moisture content in packaging materials (wood, paper)

3. Presence of air-gaps in packaging materials (corrugated fiberboard)

4. Effect of temperature, humidity and water in the environment

5. Effect of existing radio frequencies and the variability of these frequencies over time 


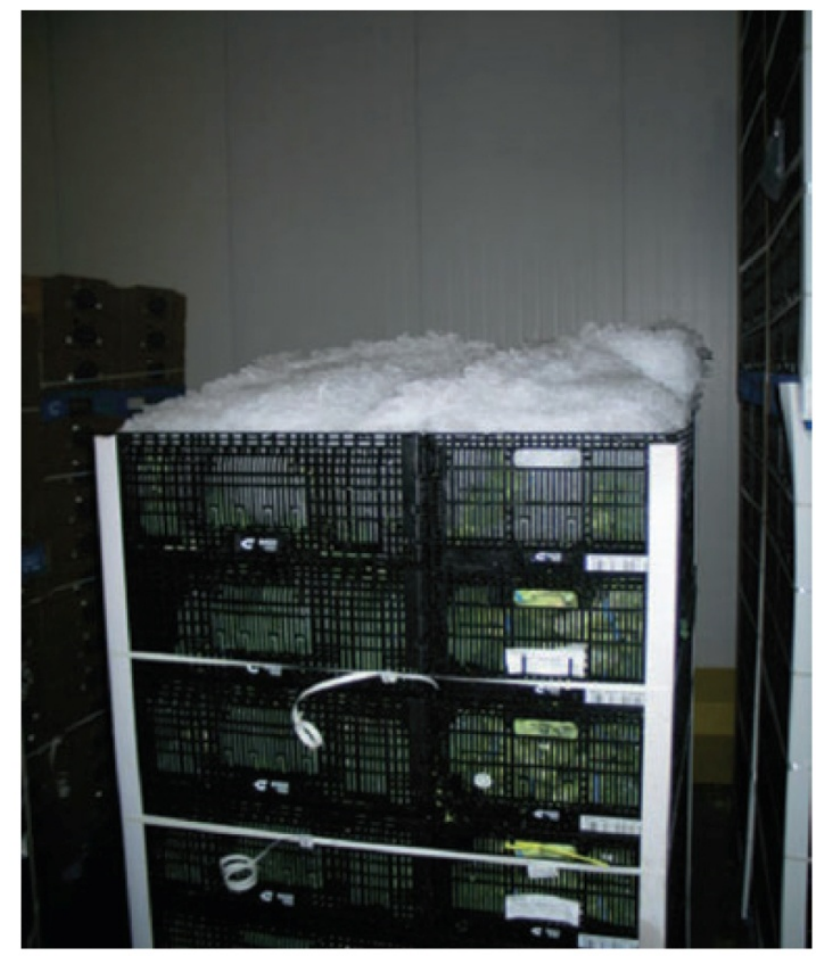

Figure 9.Testing of RPCS with ice slurry cooling of produce.

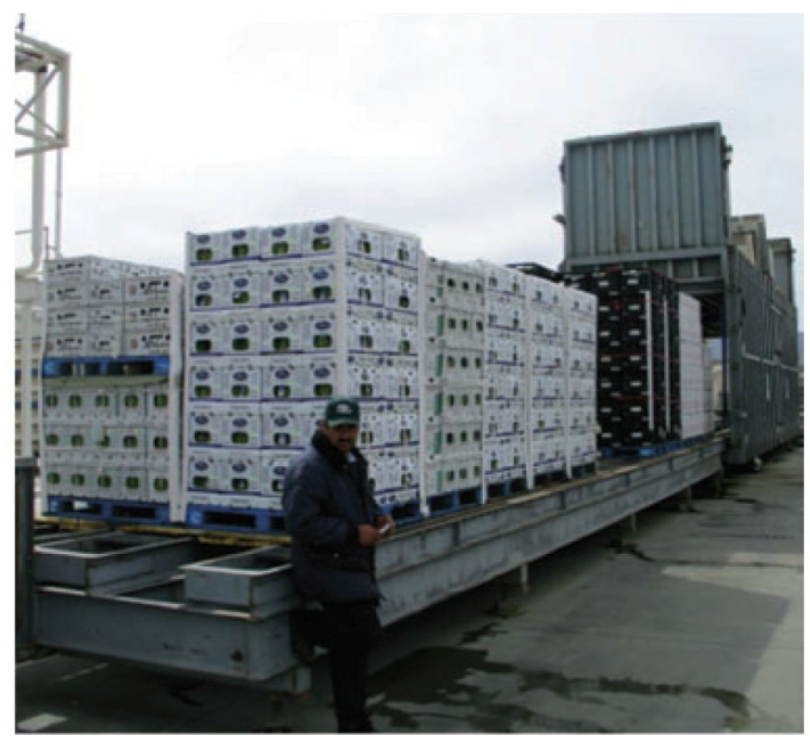

Figure 10. Hydra cooling of RFID-tagged corrugated boxes. 


\section{CONCLUSION}

The supply chain is increasingly being viewed as a key competitive component for all organizations. A supply chain consists of multi-tiers of suppliers, manufacturers and distributors. As materials flow from the supplier to the end costumer, value and costs are consistently added. As the finished product moves towards a retailer, the supply chain becomes more intricate with thousands of products sourced from thousands of suppliers. At the retail stores, such factors as out-of-place or out-of stock items play a critical role in the stores' overall profitability.

RFID is a technology that holds the promise of providing end-to-end real-time visibility of all items moving through the supply chain. A relatively new identification technology, if implemented appropriately, it could help track inventory to improve the supply chain efficiency and reduce shrinkage due to missing or undelivered inventory. RFID also holds the capability to help decrease counterfeit products from the market, to identify and efficiently recall outdated or tainted products, etc. Despite the promises, this immature technology has its challenges such as global standardization, cost and privacy issues.

This paper offers insights into several leading companies' efforts towards implementing this technology. Based on all the pilots and reviews reported in this paper, the authors have found that testing and evaluation of RFID readability and effectiveness is complex and critical due to the wide range of interactions and factors that can influence tag read based on the packaging, product, environment and material-handling equipment. Therefore, each product and package needs to be evaluated based on its anticipated use environment to provide high accuracy of reads in the shortest time and minimum duplication of equipment. ${ }^{19}$

\section{REFERENCES}

1. Overby CS, Walker J, Wilson CP. RFID at What Cost? What Wal-Mart Compliance Really Means. Forrester Research Inc., 1 March 2004, p. 7. 
2. Top 25: innovations; the Internet, ranked no. 1, changed the world. 2005; http://www.cnn.com/ 2005/TECH/01/03/cnn25.top25.innovations/ [accessed 30 September 2005].

3. RFID report provides forecasts \& analysis. 2005; http://www.usingrfid.com/news/read.asp? 1c=j2443ax354zt [accessed 25 September 2005].

4. RFID take-up set to increase in 2005. 2005; http://foodproductiondaily.com/news/newsng.asp?n=57389-rfid-take-up [accessed 4 March 2006].

5. Koprowski GJ. RFID finding new applications everywhere. 2005; http://www.technewsworld. com/story/44396.html [accessed 30 September 2006].

6. Jaques R. Global RFID market to top $\$ 7$ bn by 2008. 2005; http://www.vnunet.com/vnunet/news/2126813/global-rfidmarket-top-7bn-2008 [accessed 10 April 2007].

7. Zaino J. Wal-Mart's RFID mandates: separating fact from fiction. Information Week, 2004; http://www.informationweek.com/story/showarticle.jhtml?articlelD=47902715\&tid=5979 [accessed 14 September 2005].

8. Wills M. RFID isn't just for big companies. RFID Journal 2004; URL: http://www.rfidjournal.com/ article/articleview/930/1/82 [published 2004, accessed 4 March 2005].

9. Bednarz A. DoD pumps up RFID effort. Network World, 2004; http://findarticles.com/p/articles/miqa3649/is_200404/ai_n9404234 [accessed 17 September 2005].

10. Porter L. The Gen 2 standard: what is it, and whatdoes it mean? White Paper, Paxar Corporation, http://www.paxar.com/products/BarcodeRFID/docomants/Gen2_Standard.pdf [accessed 24 September 2005]. 
11. O'Connor MC. Impinj to ship 50 M Gen 2 chips in 2005. RFID Journal 2005; http://www.rfidjournal. com/article/articleview/1855/1/1/ [accessed 24 September 2005].

12. O'Connor MC. Philips, partners testing Gen 2 chips. RFID Journal 2005; http://www.rfidjournal.com/ article/articleview/1480/1/1/ [accessed 24 September 2005].

13. Chiesa M, Genz R, Heubler F et al. RFID - a week long survey on the technology and its potential. Research Paper, Interaction Design Institute, Ivrea, Milan, Italy, 4 March 2002.

14. Babcock B. EPC Technology in the packaging supply chain. Presentation at RFIDretail Seminar, Proceedings held at Monterey, CA by Michigan State University, East Lansing, MI, 2005.

15. Jones KC. RFID demand set to rise: report. Tech Web. 2005; http://www.techweb.com/wire/ebiz/ 171201670. [accessed 1 October 2005].

16. Roberti M. Gillette sharpens its edge. RFID Journal Magazine 2004; http://www.rfidjournal.com/ magazine/article/900 [accessed 1 October 2005].

17. Jackson J. Meeting retailer RFID challenges for apparel by VF Corporation. Presentation at RFIDretail Seminar, Proceedings held at Monterey, CA by Michigan State University, East Lansing, MI, 2005.

18. McCartney M. Keynote retail panel: meeting technology challenges and succeeding. Presentation at RFIDretail Seminar by Michigan State University, East Lansing, MI, 2005.

19. Singh SP. Challenges and opportunities for RFID implementation for retailers-apparel, consumer goods and fresh produce. International Packaging Seminar, CETEA-Brazil Packaging Institute, Sao Paulo, Brazil, May 2005. 